\title{
Multi-Model Imaging of Local Chemistry and Ferroic Properties of Hybrid Organic-Inorganic Perovskites
}

\author{
Yongtao Liu ${ }^{1,2}$, Anton V. Ievlev ${ }^{1}$, Liam Collins ${ }^{1}$, Alex Belianinov ${ }^{1}$, Songkil Kim ${ }^{3}$, Benjamin Doughty ${ }^{4}$, \\ Stephen Jesse ${ }^{1}$, Mahshid Ahmadi ${ }^{2}$, Scott T. Retterer ${ }^{1}$, Kai Xiao ${ }^{1}$, Bobby G. Sumpter ${ }^{1}$, Sergei V. \\ Kalinin ${ }^{1}$, Bin $\mathrm{Hu}^{2}$, and Olga S. Ovchinnikova ${ }^{1 *}$ \\ 1. Center for Nanophase Materials Sciences, Oak Ridge National Laboratory, Oak Ridge, TN, United \\ States \\ 2. Department of Materials Science and Engineering, University of Tennessee, Knoxville, TN, United \\ States \\ 3. School of Mechanical Engineering, Pusan National University, Busan, South Korea \\ 4. Chemical Sciences Division, Oak Ridge National Laboratory, Oak Ridge, TN, United States \\ * Corresponding author: ovchinnikovo@ornl.gov
}

Hybrid organic-inorganic perovskites (HOIPs) have shown promise in photovoltaic (PV) devices, due to their rapidly growing power conversion efficiency (PCE) and low-cost [1]. However, fundamental understandings of HOIPs PV still lags, specifically the origin of high PCE and the anomalous currentvoltage (I-V) hysteresis [2]. Although ferroelectricity is proposed to be responsible for related behavior, the presence of ferroelectricity in HOIPs is hotly debated and convincing evidence supporting ferroelectricity is missing. This is likely due to the high ionic conductivity of HOIPs, which complicates the HOIPs ferroic characterization. A clear understanding of the interplay between ferroic and ionic behavior in HOIPs will be helpful for intelligent PV design.

In this work, we use multi-modal chemical and functional imaging to unveil an interaction between ionic distribution and ferroelastic twin domains in methylammonium lead iodide $\left(\mathrm{CH}_{3} \mathrm{NH}_{3} \mathrm{PbI}_{3}\right.$ or $\left.\mathrm{MAPbI}_{3}\right)$. The ionic distribution of the $\mathrm{CH}_{3} \mathrm{NH}_{3} \mathrm{PbI}_{3}$ thin films was studied using helium ion microscopy secondary ion mass spectrometry (HIM-SIMS), which allows us to detect the local chemical composition of $\mathrm{CH}_{3} \mathrm{NH}_{3} \mathrm{PbI}_{3}$. Figure 1a is the HIM-SIMS mass spectra of $\mathrm{CH}_{3} \mathrm{NH}_{3} \mathrm{PbI}_{3}$ with $\mathrm{Ne}^{+}$as the primary ion beam; $\mathrm{CH}_{3} \mathrm{NH}_{3}{ }^{+}$and $\mathrm{Pb}^{+}$signals are labeled [3]. Figure $1 \mathrm{~b}$ shows the chemical map for $\mathrm{CH}_{3} \mathrm{NH}_{3}{ }^{+}$, where we observed the striped $\mathrm{CH}_{3} \mathrm{NH}_{3}{ }^{+}$distribution that corresponds to ferroelastic twin domains. This suggests a variation in $\mathrm{CH}_{3} \mathrm{NH}_{3}{ }^{+}$distribution in the ferroelastic twin domains. This was confirmed further by atomic force microscopy infrared spectroscopy (AFM-IR), which also yielded a chemical map with striped $\mathrm{CH}_{3} \mathrm{NH}_{3}{ }^{+}$distribution [3]. In piezoresponse force microscopy (PFM) measurements, this striped $\mathrm{CH}_{3} \mathrm{NH}_{3}{ }^{+}$distribution can translate to measurable variations of elastic moduli that act as the dominant driver for PFM measurements, leading to this twin domain exhibits 'piezoelectric-like' contrast in PFM images. Indeed, our band excitation (BE)-PFM amplitude (Figure 1c) and phase (Figure 1d) images show these twin domains even if the piezoresponse of these twin domains was proven to be below the sensitivity of PFM [3-6]. As HOIPs are well known to exhibit ionic mobility, this striped ionic distribution can also affect switching spectroscopy (SS)-PFM hysteresis measurements, due to ion migration under the probe. It is worth noting that although lateral PFM is less sensitive to ion migration under probe, this elastic driving mechanism can also affect lateral PFM measurement via friction induced cantilever torsion. In addition, we demonstrate that this correlated ferroelastic domains and $\mathrm{CH}_{3} \mathrm{NH}_{3}{ }^{+}$distribution change the interaction of the material with light through altering transition dipole moment [3]. Furthermore, temperature-driven phase transition studies reveal that the local chemical 
variation plays roles in the formation of ferroelastic twin domains, providing a pathway to manipulate ferroelastic twin domains and hence optimize optoelectronic properties of HOIPs. Overall, this work shines a light on ferroic-ionic interplay in HOIPs and offers suggestions to take advantage of ferroic properties of HOIPs in optoelectronic applications [7].

\section{References:}

[1] HJ Snaith, Nature materials 17 (2018), p. 372.

[2] HJ Snaith et al., The journal of physical chemistry letters 5 (2014), p. 1511.

[3] Y Liu et al., Nature materials 17 (2018), p. 1013.

[4] Y Liu et al., Applied Physics Letters 113 (2018), p. 072102.

[5] A Labuda and R Proksch, Applied Physics Letters 106 (2015), p. 253103.

[6] S Jesse et al., Annual review of physical chemistry 65 (2014), p. 519.

[7] Research was conducted at Oak Ridge National Laboratory's Center for Nanophase Materials Sciences (CNMS), which is a U.S. Department of Energy Office of Science User Facility. The authors acknowledge Nature materials for reusing pre-published material (Figure 1a).
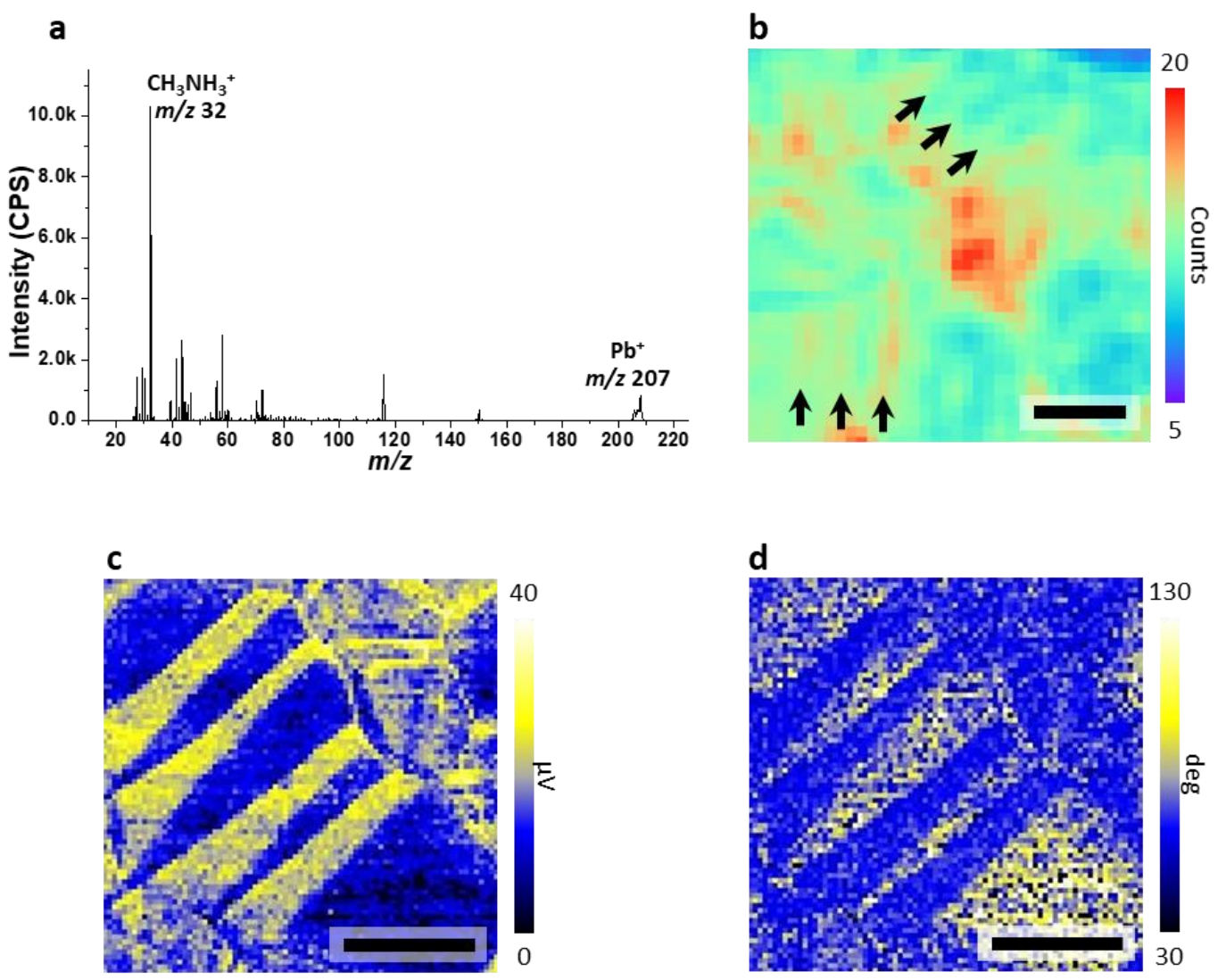

Figure 1. a, Mass spectra of HIM-SIMS measurement, $\mathrm{CH}_{3} \mathrm{NH}_{3}{ }^{+}$and $\mathrm{Pb}^{+}$signals are detected; $\mathbf{b}$, $\mathrm{CH}_{3} \mathrm{NH}_{3}{ }^{+}$distribution map shows striped $\mathrm{CH}_{3} \mathrm{NH}_{3}{ }^{+}$distribution, some striped $\mathrm{CH}_{3} \mathrm{NH}_{3}{ }^{+}$distribution is indicated by black arrows. c, Band excitation piezoresponse force microscopy (BE-PFM) amplitude map and $\mathbf{d}, \mathrm{BE}-\mathrm{PFM}$ phase map show ferroelastic twin domain (seen as stripes) in $\mathrm{CH}_{3} \mathrm{NH}_{3} \mathrm{PbI}_{3}$ thin films. Scale bars in b, $\mathbf{c}, \mathbf{d}, 1 \mu \mathrm{m}$. 